\title{
Characterization of the orthotropic viscoelastic tensor of composites using the Ultrasonic Polar Scan
}

\author{
A. Martens ${ }^{\mathrm{a}}$, M. Kersemans ${ }^{\mathrm{b}}$, J. Daemen $^{\mathrm{a}}$, E. Verboven $^{\mathrm{b}}$, W. Van Paepegem ${ }^{\mathrm{b}}$, \\ S. Delrue ${ }^{\mathrm{a}}, \mathrm{K}$. Van Den Abeele ${ }^{\mathrm{a}}$ \\ ${ }^{a}$ Wave Propagation and Signal Processing (WPSP), Department of Physics, KU Leuven - \\ Kulak, 8500 Kortrijk, Belgium \\ ${ }^{b}$ Mechanics of Materials and Structures (MMS),Department of Materials, Textiles and \\ Chemical Engineering, Ghent University, Technologiepark-Zwijnaarde 903, 9052 \\ Zwijnaarde, Belgium
}

\begin{abstract}
An enhanced two-stage inversion scheme for the determination of the viscoelastic properties of orthotropic plates (e.g. fiber reinforced polymers) based on the pulsed ultrasonic polar scan (P-UPS) is presented. A forward numerical model for the P-UPS is combined with an efficient optimizer routine (particle swarm optimizer, PSO) to inversely extract the complex orthotropic stiffness tensor, as well as the orientation of the main axes of orthotropy. The developed inversion technique is validated on synthetic data, yielding accurate results with small deviations: elasticity tensor $<0.5 \%$, viscosity tensor $<2 \%$ and main axes of orthotropy $<0.03^{\circ}$. The inversion procedure has been further applied to analyze actual experimental P-UPS recordings on an aluminum plate as well as a unidirectional $[0]_{8}$ carbon/epoxy laminate. The obtained inversion results yield complex stiffness parameters which are in close agreement to reported values in literature, and confirms the potential of the UPS technique and its inversion optimization routine as an enhanced NDT technique to quickly and accurately obtain characteristic material properties of composites.
\end{abstract}

Keywords: Material characterization, Ultrasonic Polar Scan, non-destructive testing, Particle swarm optimization, composites, orthotropy, viscoelasticity

Email address: arvid.martens@kuleuven-kulak.be (A. Martens)

Preprint submitted to Elsevier

September 10, 2019 


\section{Introduction}

In the last decades there has been a profound interest in the usage and development of high performance materials such as carbon fiber reinforced plastics (CFRP). The specific stiffness and strength of these (cross-) laminated materials are exploited in primary components of for instance airplanes, automobiles, etc. Naturally, the fiber orientation and the lay-up of these materials introduce the supplementary complexity that their material parameters become anisotropic. This turns the characterization of their stiffness parameters and the detection of possible defects in such materials into a difficult task. Several non-destructive techniques, mainly based on ultrasound wave propagation, have been developed to infer the dynamic characteristics of orthotropic materials. Most of the ultrasonic techniques are based on the propagation of bulk waves inside the plate exploiting the close relationship between elasticity and the time-of-flight (TOF) [1] 8. However, the contribution of material viscosity is often neglected during the analysis, potentially providing deceiving information on the investigated material. Aside from the omitted influence of viscosity, bulk wave based techniques suffer from several additional limitations which ultimately hampered a real breakthrough of these techniques in further research and applications:

- The bulk wave approximation poses a limitation on the frequency range that can be used during the experiments as bulk waves propagation requires the wavelength $(\lambda)$ to be small compared to the thickness $(d)$ of the plate, i.e. $\lambda \ll d$. On the other hand, there is also an upper bound on the frequency due to both damping characteristics and the compulsory condition of a homogenized structure.

- Bulk wave based techniques neglect effects arising from the plate boundaries as the bulk wave approximation assumes the plate to have an infinite thickness 9 , 10. This is particularly observed in the discontinuous phase shift of the transmitted transverse bulk wave, as seen on Figure 1, which emerges from the evanescent behavior of the longitudinal bulk wave when 


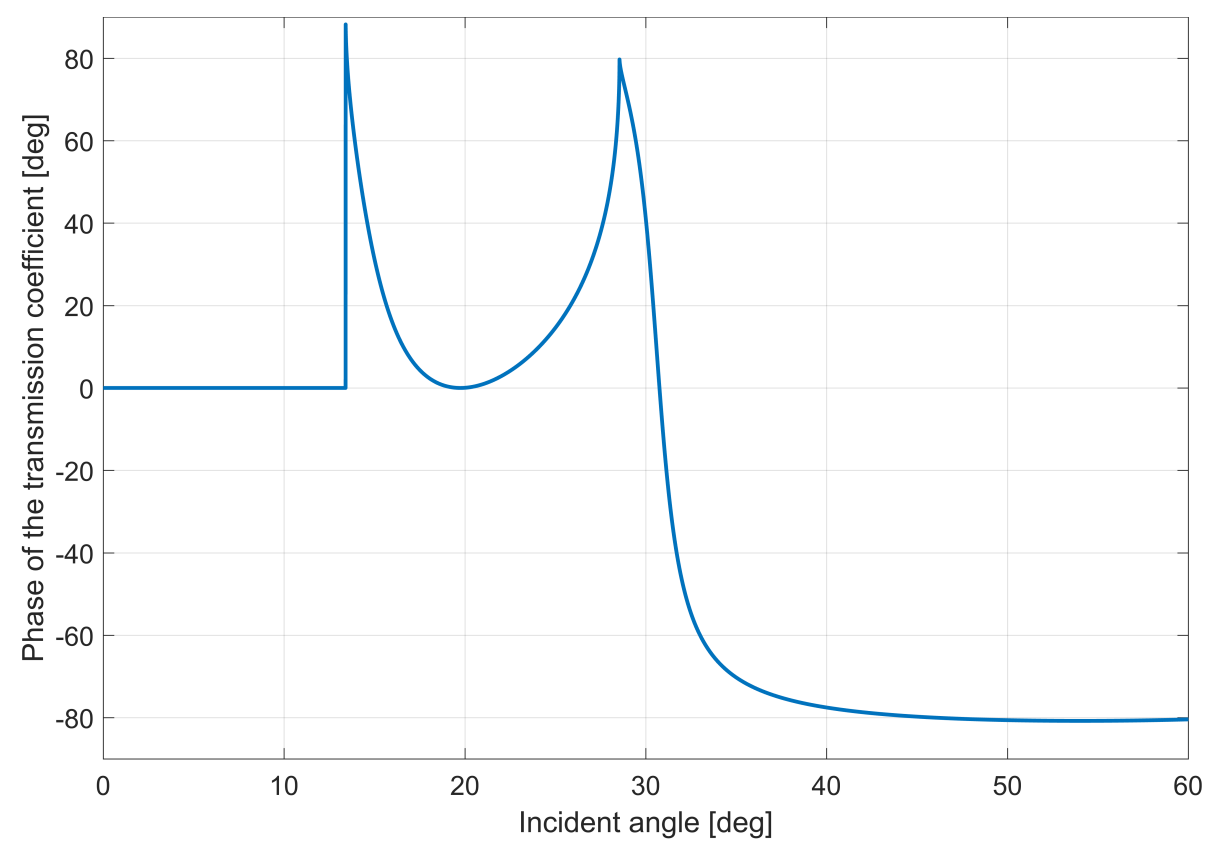

Figure 1: Numerical calculation of the transmission coefficient phase of the shear waves for an aluminum plate (material properties taken from literature $C_{11}=110 \mathrm{GPa}$ and $C_{44}=26$ GPa $\left[12\right.$. The sharp peaks correspond to the critical angles of the longitudinal $\left(\theta=13^{\circ}\right)$ and the shear wave $\left(\theta=28^{\circ}\right)$.

passing the first critical angle $\left(\theta_{\text {crit }}=13^{\circ}\right.$ for aluminum). Although independent on both the frequency and plate thickness, it does induce an additional time-delay which should be accounted for when using TOF data to infer the elasticity constants [10, 11].

- The bulk wave technique requires a-priori knowledge of the investigated material as the inversion of the data is based on measurements along certain known in-plane angles. In an earlier publication, we have shown that a mismatch of only a few degrees already results in large errors on the subsequently inverted elasticity constants [10]. Hence, those methods a-priori need to know the exact symmetry class and main symmetry orientations of the investigated material.

The Ultrasonic Polar Scan (UPS) resolves the above mentioned limitations, and 
has the potential to characterize the full viscoelastic tensor of orthotropic media [13 15] without prior knowledge of the symmetry orientation. Moreover, knowledge on the full tensor also offers the potential to detect damage in the material, as any kind of microscopic damage in CFRP induces discernable changes in the macroscopic material parameters which in turn affect the UPS measurement [16. The pulsed UPS (P-UPS) technique gathers information about the viscoelastic behavior of a solid plate through insonification of a fixed material spot with a broadband ultrasonic pulse over a wide range of oblique angle pairs $\Psi(\theta, \phi)$, where $\theta$ and $\phi$ correspond to the familiar spherical coordinate angles respectively depicting the out-of plane incidence angle and the in-plane direction (fig. 2a). At each angle pair, the transmitted signal is captured resulting in a vast amount of information on the investigated sample. These recorded time signals are simultaneously analyzed to extract the maximum amplitude (amplitude P-UPS) and the associated time instance (TOF P-UPS). The subsequent mapping of these features on a polar diagram results in a local fingerprint of the investigated sample. Straight lines (or spokes) through the center correspond to a constant in-plane angle $\phi$ and variable incidence angle $\theta$, whereas concentric circles correspond to constant $\theta$ and variable in-plane orientation $\phi$. Figure $2 \mathrm{~b}$ displays a P-UPS recording for a unidirectional $[0]_{8}$ CFRP laminate. The PUPS image reveals characteristic patterns which relate to the material symmetry class. Obviously, for an isotropic sample the patterns would all be concentric circles. The stretched appearance shown in Figure $2 \mathrm{~b}$ reveals the transversal isotropy of the investigated sample [15]. Aside from an straightforward visual inspection of the contours, one can further analyze the actual landscape (amplitude or TOF) of the P-UPS images in more detail using a numerical model and arrive at an inversion procedure that allows the determination of the full local orthotropic viscoelastic tensor.

The objective of the present study is to illustrate the performance of an advanced characterization procedure based on a particle swarm optimization (PSO) approach to extract the 18 orthotropic stiffness moduli (9 for elasticity and 9 for viscosity) from a P-UPS measurement, simultaneously with the main 


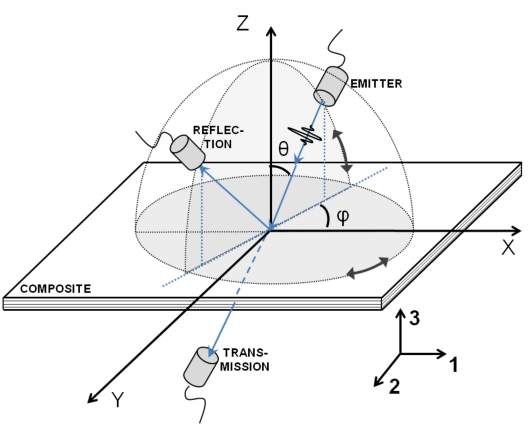

(a)

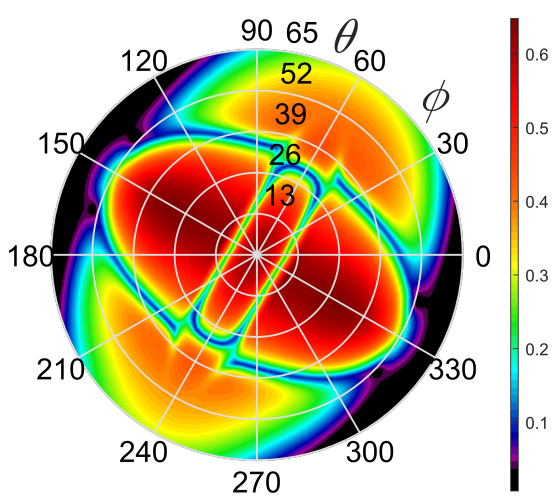

(b)

Figure 2: Illustration of the UPS (P-UPS) principle (a). Experimental pulsed UPS measurement (amplitude P-UPS) of a $\left[0^{\circ}\right]_{8}$ carbon/epoxy plate at an $f_{c} d$ value of $5 \mathrm{MHz} . \mathrm{mm}$, with a colorbar that is normalized to maximum amplitude of the incident field (b).

symmetry orientations. The exploitation of evolutionary search algorithms has already proven successful for the extraction of elastic moduli [10, 18. The inversion algorithm consists of two-stages. Stage I employs the TOF landscape (TOF P-UPS data) to extract a first guess on the elasticity constants. This first stage has already been successfully employed on both numerical simulations and experimental recordings of orthotropic media giving promising results [10]. In the present study, we extend the inversion based on the TOF landscape to also reveal the symmetry orientation at this stage. Stage II subsequently analyzes the amplitude landscape (amplitude P-UPS data) to fine-tune the elasticity constants and symmetry orientation from Stage I, and to extract the viscosity constants. After a brief introduction of the P-UPS forward model and the developed two-stage inversion approach, we will show the efficiency of the developed procedure to extract orthotropic viscoelasticity for a range of numerical and experimental test cases. 


\section{Theoretical background}

\subsection{Harmonic wave propagation in anisotropic media}

For a linear anisotropic medium one can combine Newton's second law with the general Hooke's law to arrive at the equation of motion (1), describing the general behavior of a wave propagating inside a material with density $\rho$ and elastic moduli $C_{i j k l}$.

$$
\rho \frac{\partial^{2} u_{i}}{\partial t^{2}}=C_{i j k l} \frac{\partial^{2} u_{l}}{\partial x_{j} \partial x_{k}} .
$$

Here $u_{i}$ denotes the $i$-th component of the particle displacement, and Einstein notation is used for indices $j, k$ and $l$. A general solution for the anisotropic case is not easily found. However, using the partial wave technique which assumes the solution to be a linear combination of plane waves with wave vector $\vec{K}$ and angular frequency $\omega=2 \pi f$, one can write down the wave propagation solution in a simple expression (2) where Einstein notation is used for index $m$.

$$
u_{i}=\sum_{q} U_{i q} e^{j\left(K_{m q} X_{m}-\omega t\right)} .
$$

Inserting this expression into the wave equation (1) leads to an eigenvalue problem known as the Christoffel equation (3). This equation provides a relation between the polarization vector, wave vector, frequency and stiffness tensor.

$$
\left(C_{i j k l} K_{j} K_{k}-\rho \omega^{2} \delta_{i l}\right) U_{l}=0 .
$$

Where $\delta_{i l}$ denotes the typical Kronecker-delta function and Einstein notation is used for indices $j$ and $k$. Assuming that the axes are oriented such that the $x_{3}$ direction is perpendicular to the plate surfaces, we can determine two of the three wave vector components $\left(K_{1}\right.$ and $\left.K_{2}\right)$ using Snell's law as it tells us that the parallel components of the wave vector ought to be continuous. Considering the fact that for an orthotropic material only nine of the $C_{i j k l}$ are independent, we can determine the remaining unknown component $K_{3}$ by transforming the Christoffel equation into a bi-quadratic sixth order polynomial in the $K_{3}$ component.

$$
\beta_{6}\left(K_{3}^{2}\right)^{3}+\beta_{4}\left(K_{3}^{2}\right)^{2}+\beta_{2}\left(K_{3}^{2}\right)+\beta_{0}=0 .
$$


Equation (4) represents the propagation of a set of 3 upward and 3 downward going waves, each of them containing one quasi-longitudinal and two quasitransverse waves.

During an actual P-UPS experiment, the inspected plates are immersed in water as this reduces the acoustic impedance mismatch at the interfaces and allows sound to penetrate the sample more easily. In order to determine the transmission coefficient, appropriate boundary conditions at the liquid/solid and solid/liquid interface should be defined i.e. expressing the continuity of the normal displacement $\left(u_{3}\right)$ and the normal stresses $\left(\sigma_{i 3}\right.$ for $\left.i=1,2,3\right)$ at each interface. Working out the corresponding algebra we arrive at the expression (5) for the transmission coefficient $(T)$, namely.

$$
T=\frac{i Y(A+S)}{(S+i Y)(A-i Y)} .
$$

Where $A$ and $S$ are respectively linked to the anti-symmetric and symmetric guided waves propagating inside the plate, whilst $Y$ describes the coupling between the immersing fluid and the solid plate [11, 19]. Viscosity is included in the theoretical model by considering the moduli to be complex valued, namely

$$
C_{i j k l}=C_{i j k l}^{\prime}-i C_{i j k l}^{\prime \prime}
$$

where the real part symbolizes the elasticity and the imaginary part the viscosity.

\subsection{Broadband pulsed wave propagation in anisotropic media}

The fixed frequency transmission coefficient derived in the previous section, however, is not sufficient to calculate the actual transmitted signal for a P-UPS experiment which employs a broadband input signal $I(t)$ (Fig. 3a). This can be resolved by calculating the inverse Fourier transform of the incident spectrum $\tilde{I}(\omega)$ (Fig. 3b multiplied by the frequency dependent transmission coefficient for each specific incidence angle $\Psi(\theta, \phi)$ :

$$
T\left(\theta, \phi, C_{i j k l}, t\right)=\int_{-\infty}^{\infty} \tilde{I}(\omega) T\left(\theta, \phi, C_{i j k l}, \omega\right) e^{i \omega t} \mathrm{~d} \omega
$$

The integral expression (6) gives us the transmission signal in time domain 


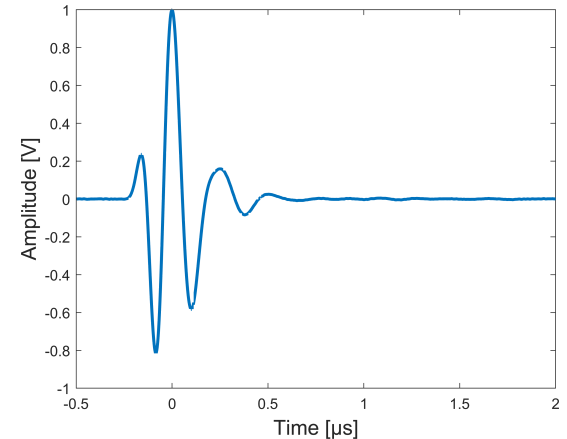

(a)

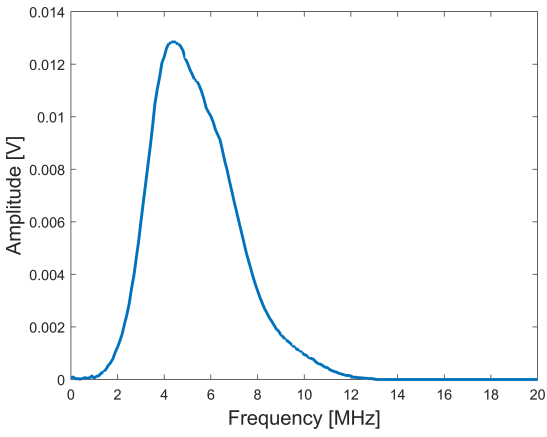

(b)

Figure 3: Time signal (a) and frequency content (b)of the ultrasonic pulse delivered by a H5K transducer (GE) during the experimental P-UPS recordings (center frequency $f_{c}=4 \mathrm{MHz}$ ).

which can be further analyzed as function of $\Psi(\theta, \phi)$. By evaluating the maximum amplitude of this signal at each angle, we obtain the amplitude P-UPS. Likewise, the TOF P-UPS is retrieved by mapping the time delay associated to this maximum amplitude with respect to a reference case where the plate is absent. However, a small time correction (Eq. (7)) needs to be introduced as the path $\left(D_{f}\right)$ to the receiving transducer beneath the plate is longer (Fig. 4a) than the radius $(R)$ of the hemisphere:

$$
t_{c o r r}=\frac{d}{V_{f}}(1-\cos \theta) .
$$

Here, $d$ is the thickness of the material plate, $V_{f}$ the speed of sound in the bounding fluid and $\theta$ the incident angle. An example illustrating the necessity of the correction term can be found in Figure $4 \mathrm{~b}$ where a spoke (a cross section at constant $\phi$ angle) of a P-UPS experiment on an aluminum plate is compared with simulation results obtained with and without correction. It is obvious that the introduction of the time correction significantly improves the agreement between simulation and experiment, especially for large incident angles $\theta$.

The forward model is easily implementable on GPU architectures using Mathworks MATLAB and takes less than 1 second to calculate both the TOF and amplitude P-UPS for a plate consisting of a single anisotropic layer taking into account 60000 angle combinations $\Psi(\theta, \phi)$. 


\section{Two-stage characterization procedure}

Inversion procedure. With a suitable and fast assessable forward wave propagation model for the P-UPS available, an optimization procedure can now be introduced in order to determine the full set of orthotropic viscoelastic tensor coefficients of the solid plate in an inverse way. We propose a two-stage algorithm in which both the TOF P-UPS and the amplitude P-UPS data is employed to obtain our premise(Fig. 5).

In stage I, the TOF data is used as a means to extract the elastic constants [10. Indeed, TOF information is closely linked to the phase velocity of the traveling waves which, to a large extent, is determined by the material elasticity. In the general case of orthotropic materials, nine coefficients of elasticity can be determined in this way. For the viscosity coefficients during stage I calculation, a random percentage $(0.1 \%-15 \%)$ of the elasticity is used such that the weak influence of viscosity on TOF data is captured to some extent. There is no optimization of the viscosity coefficients at this stage.

In stage II, the amplitude P-UPS data is used to infer the unknown viscosity

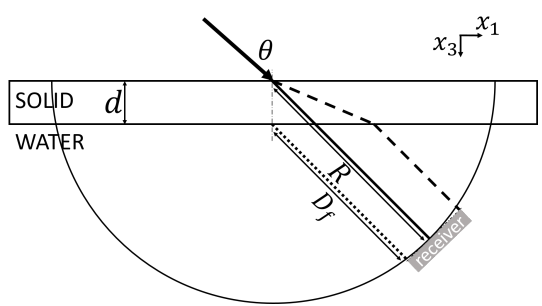

(a)

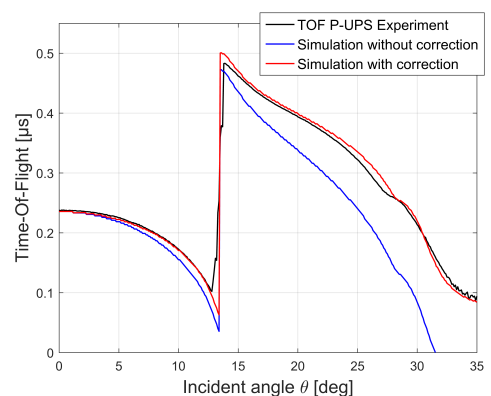

(b)

Figure 4: (a) Cross-section of the lower hemisphere (radius $R$ ) of the UPS technique along a fixed in plane angle showing the ray path of a wave with incidence angle $\theta$. The dashed and dotted line correspond respectively to the physical path and the path $\left(D_{f}\right)$ of the simulated wave. (b) Cross-section of an aluminum TOF P-UPS experiment at a certain in-plane angle $\phi$ : experimental recording (black), corrected simulation result (red) and uncorrected simulation result (blue). 


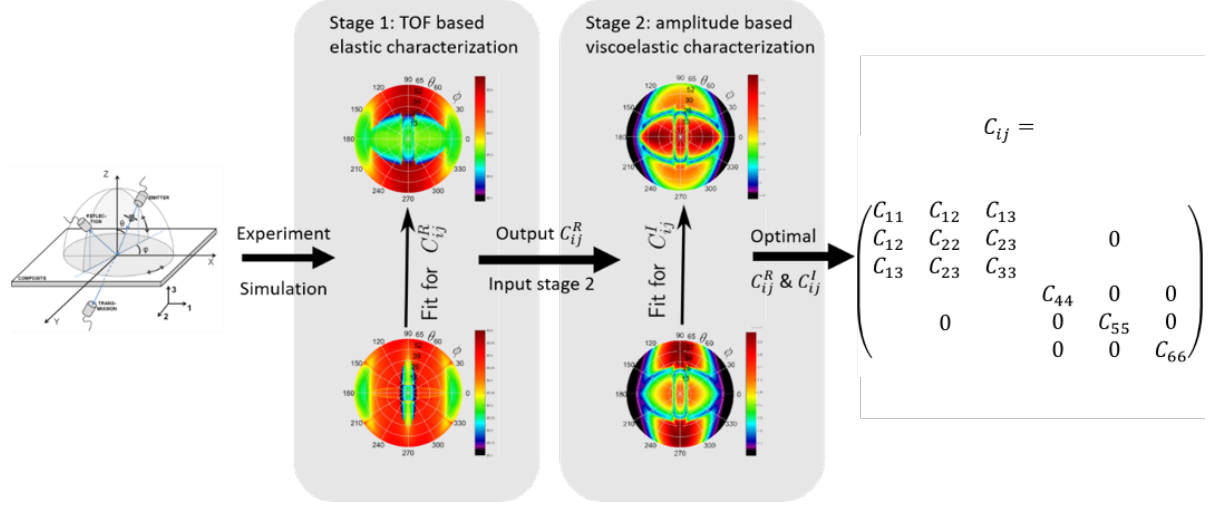

Figure 5: Schematic of the developed two-stage inversion procedure. The first stage fits the TOF P-UPS landscape to retrieve a best guess on the nine elasticity constants of the investigated sample. The second stage uses the available amplitude P-UPS data combined with the best guess results of stage one to determine the full set of the viscoelastic tensor coefficients.

parameters. Indeed, amplitude data is particularly useful for the determination of the viscosity parameters as the amplitude level of the transmitted waves is determined by their energy loss when traveling through the plate and as such provides a good representation for the material damping. The optimized elastic parameters of stage I are used as input for the elasticity in stage II. This 'best guess' assumption ensures that we capture the weak coupling that exists between viscosity and elasticity in amplitude data. Hence, our 9-dimensional search space (elastic tensor) of stage I is expanded to an 18-dimensional search space (elastic and viscous tensor). In both stages of the inversion procedure a least-squares function is evaluated measuring the difference between experiment and simulation for a large set of randomly chosen incidence angles $\Psi(\theta, \phi)$ simultaneously. The goal is to minimize this difference within the parameter search space. As a benefit, the large number of target data points, randomized within a polar plot, eliminates the need for a priori knowledge on the symmetry class of the inspected material. Hence, an additional parameter $\phi_{e}$, representing the main symmetry orientation angle is added to the search space totaling for 10 (stage I) or 19 (stage II) parameters to be determined. 
Optimization algorithm. As a general orthotropic viscoelastic tensor has 9(18) independent variables. Hence an efficient algorithm exploring this high dimensional search space is required to accomplish the optimization [20]. Here, we adopted the particle swarm optimization (PSO) approach for this task. Generally speaking, a PSO algorithm solves the minimization procedure by imitating the common behavior of a flock of birds searching for food, where the positions of the feed correspond to the minima of the function, and the amount of food is associated to the depth of the minimum. Initially, the flock scatters over the whole domain searching for food. When some birds find spots with more food, the other birds will adjust their position accordingly. In the end, the whole flock will congregate around the spot with the most food (i.e. the global minimum of the function is retrieved). As a PSO algorithm is stochastic, a statistical analysis on multiple runs of the inversion procedure needs to be performed in order to retrieve a correct set of material properties. For each of the test cases discussed in this study, the inversion procedure has been run 100 times, and relevant statistics have been applied on the output of these runs.

Inversion parameters. The initial set-up of the inversion procedure requires several model parameters to be supplied. First a rough estimate of the search space needs to be provided. For instance, for the synthetic P-UPS test-case using simulated data, we assume that the search space is bounded for the elasticity tensor to $\pm 60 \%$ of the actual values. In addition, the viscous part is estimated to lay between $0.1 \%$ and $15 \%$ of the elasticity tensor for all of our test-cases. This is a reasonable assumption as most materials considered in our studies do not exceed these percentages for the viscosity. The last search space parameter is the deviation angle $\phi_{e}$ for which the bounds are set to $-80^{\circ}$ and $80^{\circ}$. However, due to the orthotropic symmetry, the in-plane angle $\phi$ can be constrained between $0^{\circ}$ and $90^{\circ}$. At the start of stage II, the mean value of the optimized elasticity parameters from stage I (over 100 runs) is used as input. The search space for the elasticity parameters in stage II is then automatically taken equal to twice 


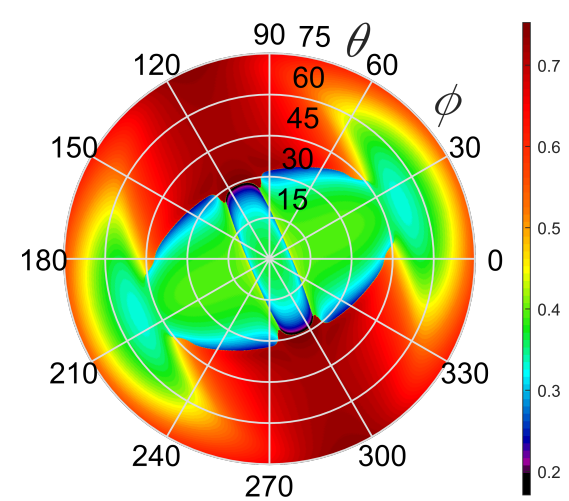

(a)

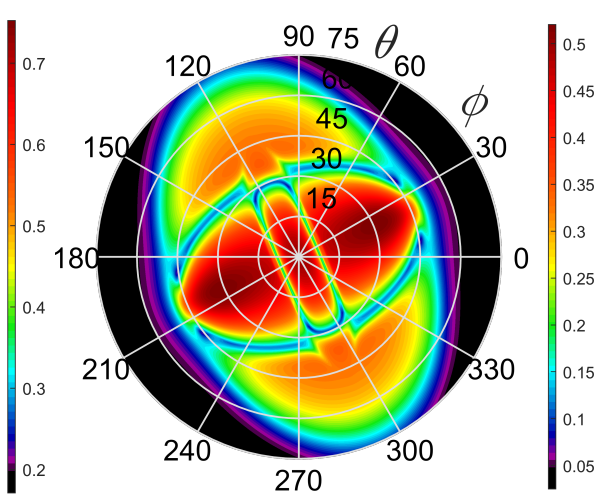

(b)

Figure 6: TOF P-UPS plot(a) and amplitude P-UPS plot(b) corresponding to a simulation for an artificial unidirectional plate $(d=1.1 \mathrm{~mm})$. The TOF colorbar is displayed in $\mu$ s, whereas the amplitude colorbar is normalized to the maximum amplitude of the input signal.

the standard deviation of the optimized elasticity parameters from stage I, i.e.

$$
C_{\text {stageII }}^{\prime}=\operatorname{mean}\left(C_{\text {stageI }}^{\prime}\right) \pm 2 \operatorname{std}\left(C_{\text {stageI }}^{\prime}\right)
$$

Besides the search space bounds, the only other parameters to be defined are: the inversion stop criteria, the number of random incidence angles $(\Psi)$ to be randomly chosen, the number of individuals used during a single run and the number of separate inversion runs. In the presently used implementation, the stop criteria during the test-cases requires the inversion run to terminate when either no change in the least-square value is detected for at least 20 iterations or when the maximum number of 200 iterations is exceeded. The number of individuals is set to 600 , the number of randomly chosen incidence angles is fixed at 3000 for synthetic data and 6000 for experimental recordings.

\section{Results and discussion}

\subsection{Characterization for an orthotropic plate based on synthetic P-UPS data}

In order to test the performance of the newly developed two-stage inversion scheme, we simulate P-UPS data for a synthetic orthotropic material ( $\rho=$ $1528 \mathrm{~kg} / \mathrm{m}^{3}, d=1.1 \mathrm{~mm}$ ) with known viscoelastic parameters (Table 1) upon 


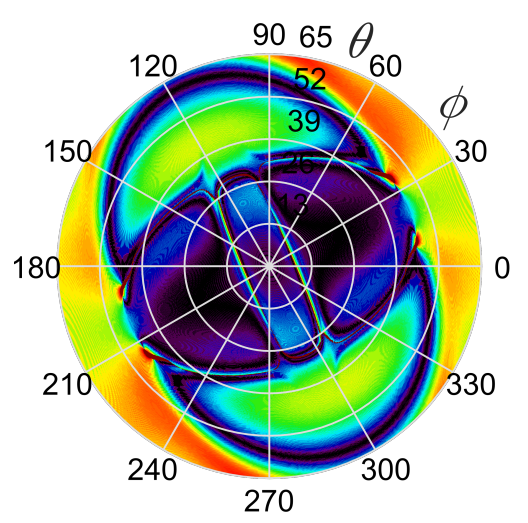

(a)

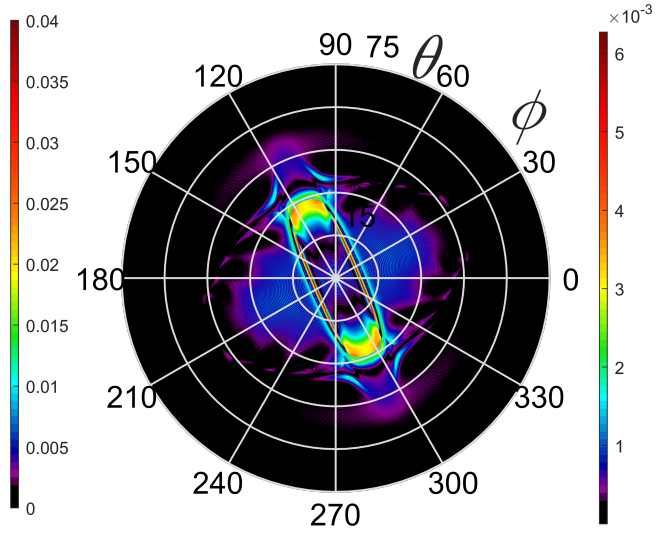

(b)

Figure 7: Difference TOF P-UPS plot (a) and difference amplitude P-UPS plot (b) displaying the absolute value of the difference between the forward P-UPS simulation based on the actual viscoelastic constants and the simulation using the inverted elastic and viscoelastic constants of respectively stage I and II. The colorbar of the TOF differences is expressed in $\mu$ s. The amplitude differences are expressed in $\mathrm{V}$, where $1 \mathrm{~V}$ refers to the maximum amplitude value of the signal.

which the inversion procedure is performed. For the forward simulation an incidence angle grid $\psi(\theta, \phi)$ with $\theta$ going from $0^{\circ}$ to $75^{\circ}$ in steps of $0.1^{\circ}$ and $\phi$ ranging from $0^{\circ}$ to $360^{\circ}$ in $0.5^{\circ}$ angle steps is chosen. In addition to the targeted viscoelastic properties we have added an in-plane deviation angle $\phi_{e}$ of $23^{\circ}$ such that ultimately an inversion search space consisting of 19 dimensions needs to be explored. The input time-signal for the P-UPS simulation is chosen to be the same signal used during an actual P-UPS experiment (Fig. 3) and resembles a Gaussian damped cosine. Figure 6 displays the simulated P-UPS data for both the TOF landscape and the amplitude landscape. During the inversion procedure of synthetic data we thoughtfully avoid the problem of the 'inverse crime' 21 by adding a random value between $\left(-0.1^{\circ}\right.$ and $\left.0.1^{\circ}\right)$ to the incidence angles and to the input signal used during the inversion. 
Table 1: PSO inversion results of stage I and II based on a numerically simulated P-UPS dataset for a plate with an orthotropic nature, density $1528 \mathrm{~kg} / \mathrm{m}^{3}$ and thickness $1.1 \mathrm{~mm}$. The statistics (mean and standard deviation) were performed using the output on 100 individual PSO runs.

\begin{tabular}{lllll|ll}
\hline & Actual values & Output stage I & Deviation & Output stage II & Deviation \\
\hline$C_{11}$ & $C_{11}^{\prime}$ & 122.73 & $130.89( \pm 23.33)$ & $6.65 \%$ & $122.64( \pm 1.53)$ & $0.07 \%$ \\
$(\mathrm{GPa})$ & $C_{11}^{\prime \prime}$ & 8.59 & $/$ & $/$ & $8.77( \pm 1.23)$ & $2.14 \%$ \\
$C_{12}$ & $C_{12}^{\prime \prime}$ & 6.57 & $6.50( \pm 2.03)$ & $1.03 \%$ & $6.42( \pm 0.79)$ & $2.26 \%$ \\
$(\mathrm{GPa})$ & $C_{12}^{\prime \prime}$ & 0.46 & $/$ & $/$ & $0.40( \pm 0.20)$ & $12.81 \%$ \\
$C_{13}$ & $C_{13}^{\prime}$ & 6.57 & $6.27( \pm 1.83)$ & $4.58 \%$ & $6.49( \pm 0.70)$ & $1.14 \%$ \\
$(\mathrm{GPa})$ & $C_{13}^{\prime \prime}$ & 0.46 & $/$ & $/$ & $0.46( \pm 0.02)$ & $0.18 \%$ \\
$C_{22}$ & $C_{22}^{\prime}$ & 13.47 & $13.60( \pm 1.50)$ & $1.03 \%$ & $13.45( \pm 0.18)$ & $0.13 \%$ \\
$(\mathrm{GPa})$ & $C_{22}^{\prime \prime}$ & 0.94 & $/$ & $/$ & $0.92( \pm 0.06)$ & $1.90 \%$ \\
$C_{23}$ & $C_{23}^{\prime}$ & 6.55 & $6.30( \pm 1.02)$ & $3.86 \%$ & $6.55( \pm 0.15)$ & $0.12 \%$ \\
$(\mathrm{GPa})$ & $C_{23}^{\prime \prime}$ & 0.46 & $/$ & $/$ & $0.45( \pm 0.04)$ & $1.70 \%$ \\
$C_{33}$ & $C_{33}^{\prime}$ & 13.47 & $13.40( \pm 1.20)$ & $0.51 \%$ & $13.47( \pm 0.20)$ & $0.07 \%$ \\
$(\mathrm{GPa})$ & $C_{33}^{\prime \prime}$ & 0.94 & $/$ & $/$ & $0.95( \pm 0.01)$ & $0.29 \%$ \\
$C_{44}$ & $C_{44}^{\prime \prime}$ & 3.40 & $3.17( \pm 0.40)$ & $6.64 \%$ & $3.40( \pm 0.01)$ & $0.04 \%$ \\
$(\mathrm{GPa})$ & $C_{44}^{\prime \prime}$ & 0.24 & $/$ & $/$ & $0.24( \pm 0.01)$ & $0.64 \%$ \\
$C_{55}$ & $C_{55}^{\prime \prime}$ & 5.86 & $5.77( \pm 0.51)$ & $1.55 \%$ & $5.86( \pm 0.01)$ & $0.04 \%$ \\
$(\mathrm{GPa})$ & $C_{55}^{\prime \prime}$ & 0.41 & $/$ & $/$ & $0.41( \pm 0.01)$ & $0.03 \%$ \\
$C_{66}$ & $C_{66}^{\prime \prime}$ & 6.25 & $6.27( \pm 0.41)$ & $0.38 \%$ & $6.25( \pm 0.01)$ & $0.03 \%$ \\
$(\mathrm{GPa})$ & $C_{66}^{\prime \prime}$ & 0.44 & $/$ & $/$ & $0.44( \pm 0.01)$ & $0.09 \%$ \\
$\phi_{e}$ & $/$ & $23.00^{\circ}$ & $22.57^{\circ}\left( \pm 1.55^{\circ}\right)$ & $0.43^{\circ}$ & $23.01^{\circ}\left( \pm 0.03^{\circ}\right)$ & $0.01^{\circ}$ \\
\hline & & & & & &
\end{tabular}

Stage I: inversion of the elastic tensor

The inversion results for stage I, using only the TOF landscape as input, are represented in column 4 and 5 of Table 1 The values of Stage I reveal that acceptable results (max. 6\% deviation) are obtained. Furthermore, a deviation angle of $22.57 \pm 1.55^{\circ}$ is retrieved which is in good agreement with the 
actual targeted value of $23^{\circ}$. The quality of the inversion results is graphically supported by the difference TOF P-UPS plot in Figure $7 \mathrm{a}$ which displays the absolute difference between the TOF P-UPS data computed with the known stiffness tensor and the ones computed with the inverted stiffness tensor at stage I. We notice that the difference is quite low $(<0.02 \mu s)$ except for the regions around the characteristic contours. These deviation can be attributed to the fact that the contours in TOF data are sharp discontinuities which are hard to fit as small changes in C-tensor values will induce large errors on the location of these contours.

Stage II: inversion of the viscoelastic tensor

The results of stage II are given in the last two columns of Table 1 and visualized in Figure 7. An excellent agreement for the elasticity part is found as most deviations are well below $0.5 \%$, only the $C_{12}$ and $C_{13}$ have deviations around $1-2 \%$ which can be attributed to the lower sensitivity of these parameters to PUPS measurements in the 1-2 plane. This is also noticeable in the deviations of the viscosity as the imaginary part of $C_{12}$ exhibits a $12.81 \%$ deviation whereas the other viscous properties have deviations below $2 \%$. In addition to the excellent results for the complex stiffness tensor, an in-plane angle of $23.01 \pm 0.03^{\circ}$ is found which is fully in agreement with the input value. The difference amplitude P-UPS plot (Fig. 7b) shows absolute error deviations well below $0.002 \mathrm{~V}$ for the majority of the incidence angles $\Psi$. Higher errors $(>0.006 \mathrm{~V})$ are only noticed close to the characteristic contours which is again due to the fact that the contours are very constricted, and as such yield large errors for small deviations in the C-tensor.

\subsection{Characterization for an aluminum plate based on real experimental P-UPS data}

In this second subsection, we employ the inversion procedure on the experimental P-UPS recording (input signal of Fig. 3a) for an aluminum plate with

thickness $d=1.500 \pm 0.002 \mathrm{~mm}$ and density $\rho=2700 \mathrm{~kg} / \mathrm{m}^{3}$. The appearance of 


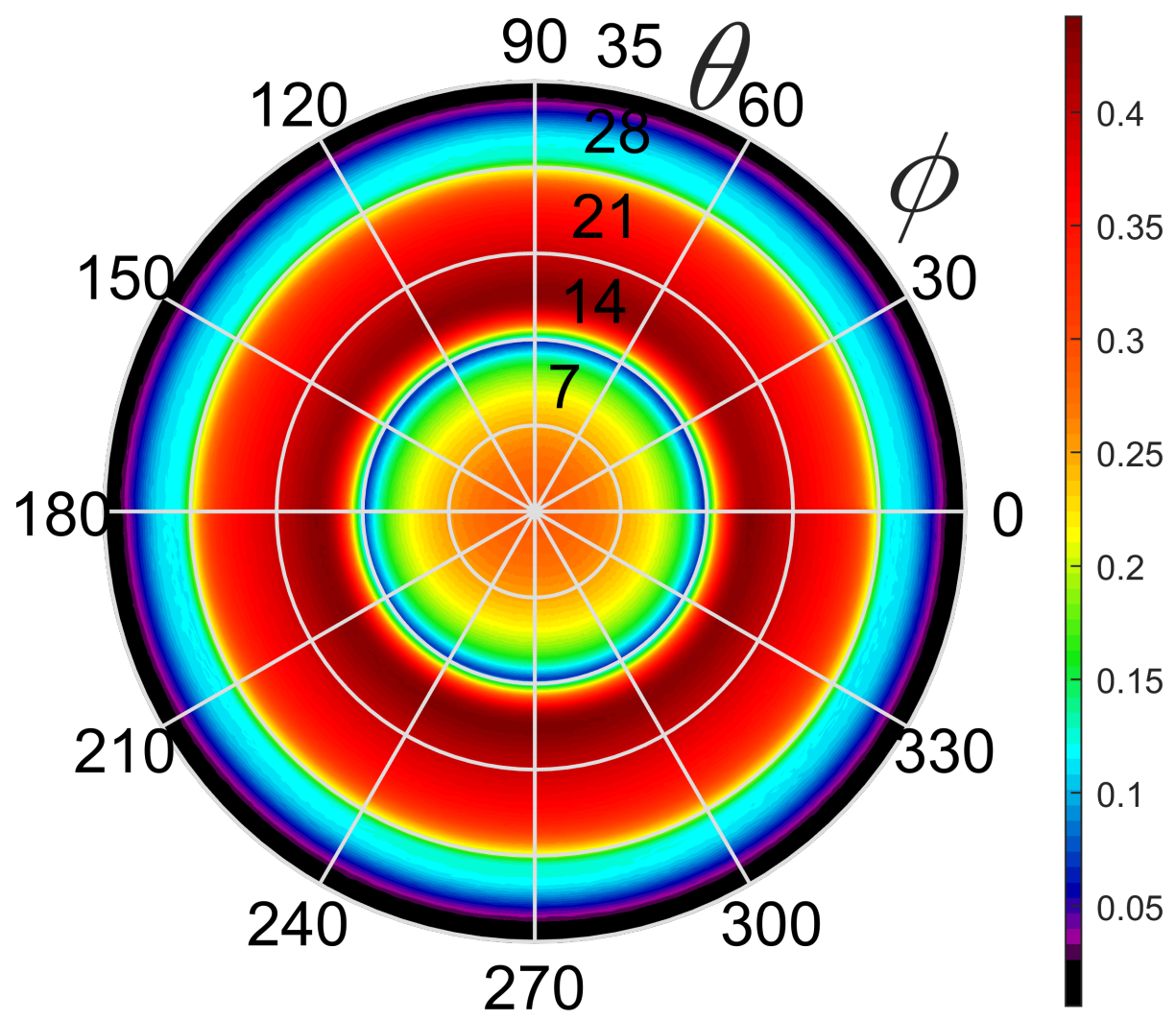

Figure 8: Experimentally obtained amplitude P-UPS plot for an aluminum plate of thickness $d=1.500 \pm 0.002 \mathrm{~mm}$ and density $\rho=2700 \mathrm{~kg} / \mathrm{m}^{3}$. Note the circular patterns suggesting an isotropic symmetry.

circular patterns on the experimental amplitude P-UPS plot in Figure 8 suggests that the aluminum sample has an isotropic symmetry. This makes aluminum a good test-case to investigate the influence of material symmetry class knowledge on the outcome of the two-stage characterization technique. Hence, we apply the inversion procedure once with the suggested symmetry class (i.e. isotropy) and once without a priori knowledge on the symmetry class (i.e. orthotropy)

\section{Stage I: inversion of the elastic tensor}

During the inversion, regardless of the symmetry class assumption, the elasticity search space is bounded at $\pm 60 \%$ of the literature values of aluminum 
$\left(C_{11=22=33}=110 \mathrm{GPa}, C_{44=55=66}=26 \mathrm{GPa}\right.$, and thus $\left.C_{12=23=13}=58 \mathrm{GPa}\right)$ [12. In the isotropic case, no deviation angle is set as this parameter contains no information. When postulating isotropy, we notice that the inverted results (column 3 of table 2) are accurately determined with very narrow error margins (standard deviations) and mean values that are in excellent agreement with the elasticity values found in literature. Ignoring the isotropic assumption in the inversion procedure (i.e. postulating general orthotropic symmetry) gives results (column 5, Table 2), which are comparable to the results obtained using the isotropic assumption and to the literature values. Note however that there is an increased standard deviation on the inverted coefficients and that $C_{33}$ shows the largest discrepancy between postulated isotropic or orthotropic symmetry inversion. Overall, these inferred values indicate that the aluminum sample indeed has an approximate isotropic nature which is even further evidenced by the universal elastic anisotropic coefficient $A^{U}$ [22] which equals 0.0283 , where a value of zero corresponds to isotropic symmetry. In addition, the deviation angle value resulting from the orthotropic symmetry inversion provides additional foundation to the isotropic symmetry statement as the large standard deviation on $\phi_{e}$ suggests a wide range of potentially valid orientations of the experimental reference frame which evidently can only be the case whenever isotropic symmetry is present.

\section{Stage II: inversion of the viscoelastic tensor}

The results of stage II, presented in columns 4 and 6 of Table 2 , indicate that both assumptions (isotropic and orthotropic) are able to successfully extract the literature elasticity values of an aluminum material from the experimental polar plot data. The difference plots for both assumptions are shown in Figures 9a 9b. Again, evidence for the isotropic material symmetry class is recovered in the orthotropic case as $A^{U}=0.013$. This is further supported by the large error margin on the deviation angle. The viscosity values (imaginary C-tensor parts) are within reasonable bounds for an aluminum plate $(0.1 \%$ to $2 \%$ of the real C-tensor parts) which is known to be a basically low-viscous material 


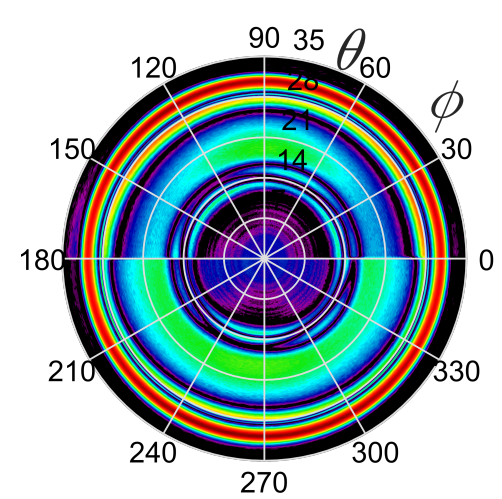

(a)

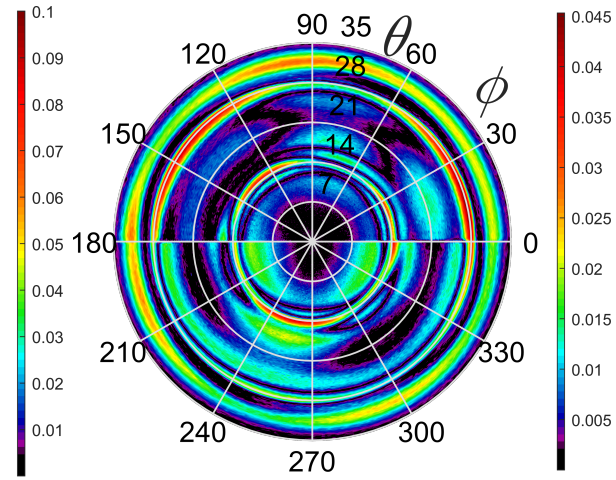

(b)

Figure 9: Difference amplitude P-UPS between the experimental polar plot for an aluminum plate $(d=1.500 \pm 0.002 \mathrm{~mm})$ and the forward simulations based on the results of the inversion procedure for the isotropic (a) and orthotropic (b) cases. The colorbar is normalized to the maximum amplitude of the input signal.

[23. However, note that the isotropic symmetry is broken in the viscous tensor ( $A^{U}=3.17$ ) of the orthotropic assumption as the extremely low value of the imaginary part of $C_{33}$ does not align with the more elevated viscosity values of $C_{11}$ and $C_{22}$. This deviation can potentially be attributed to the rolling process during manufacturing of the aluminum sheet which may have altered the viscosity in the 3-direction. The inferred deviation from isotropy for the viscosity is masked in the amplitude P-UPS image (Fig. 8) as the inverted viscosity tensor is close to the one of a transverse isotropic material with the isotropy plane coinciding with the 1-2 plane. Hence, experiments are effectively done in the isotropy plane of the material. The large standard deviations on the elastic $C_{12}$ and $C_{66}$ parameters in the orthotropic case can be attributed to the lower sensitivity of the inversion procedure towards these parameters. This results from the fact that $C_{12}$ and $C_{66}$ are mainly linked to the propagation of shear horizontal waves which cannot be excited by the P-UPS technique for a (quasi-) isotropic material. Upon closer inspection of the difference amplitude P-UPS (Fig. 9), the best result is found when assuming general orthotropic symmetry for the inversion as the overall difference $(<0.0057 \mathrm{~V})$ is much lower 


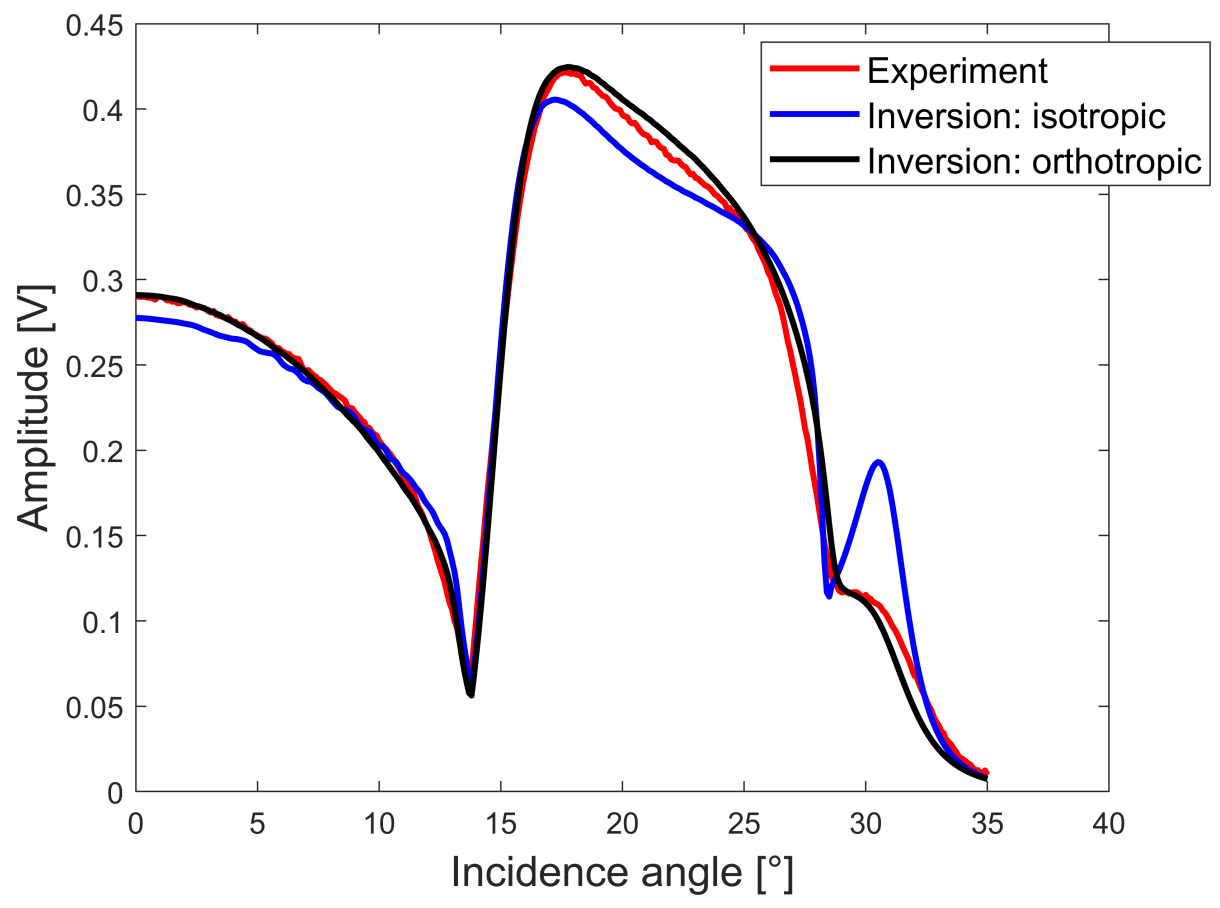

Figure 10: Cross-section ( $\phi=0$ ) of the TOF P-UPS experiment (red) for an aluminum plate of thickness $d=1.500 \pm 0.002 \mathrm{~mm}$, together with the results of stage I of the inversion procedure for respectively the isotropic (black) and the orthotropic symmetry assumption (blue).

than in the isotropic assumption $(<0.011 \mathrm{~V})$. Most of the deviations between experiment and inversions for both cases can be located in the vicinity of the characteristic contours. In addition to the difficulty of exactly matching the TOF discontinuities and amplitude extrema, as discussed earlier in the first subsection, there is also a contribution to the errors in this zone which are attributed to the fact that the UPS forward model assumes plane waves and does not take into account the integrating effect resulting from the finite size of the used H5K transducer.

The better match of the inversion based on the orthotropic symmetry postulation can be attributed to the larger search space that corresponds to this assumption (19 parameters) allowing a greater flexibility to the algorithm to adjust for any small divergence from a true isotropic symmetry, in either elastic or 
Table 2: Inversion results for both stages of a P-UPS experiment performed on an aluminum plate of thickness $d=1.500 \pm 0.002 \mathrm{~mm}$ and density $\rho=2700 \mathrm{~kg} / \mathrm{m}^{3}$ assuming either an apriori isotropic or general orthotropic symmetry. Note, the excellent agreement with literature values $\left(C_{11=22=33}=110 \mathrm{GPa}, C_{44=55}=66=26 \mathrm{GPa}, C_{12=13=23}=58 \mathrm{GPa}\right)$.

\begin{tabular}{llllll}
\hline & \multicolumn{3}{c}{ isotropic assumption } & \multicolumn{2}{c}{ orthotropic assumption } \\
& & Output stage I & Output stage II & Output stage I & Output stage II \\
\hline$C_{11}$ & $C_{11}^{\prime}$ & $109.31( \pm 3.04)$ & $108.13( \pm 1.45)$ & $114.23( \pm 25.76)$ & $108.56( \pm 4.10)$ \\
$(\mathrm{GPa})$ & $C_{11}^{\prime \prime}$ & $/$ & $1.72( \pm 0.28)$ & $/$ & $3.21( \pm 1.02)$ \\
$C_{12}$ & $C_{12}^{\prime}$ & $56.53( \pm 9.93)$ & $56.12( \pm 1.47)$ & $51.68( \pm 18.26)$ & $52.39( \pm 25.73)$ \\
$(\mathrm{GPa})$ & $C_{12}^{\prime \prime}$ & $/$ & $1.25( \pm 0.55)$ & $/$ & $1.12( \pm 1.06)$ \\
$C_{13}$ & $C_{13}^{\prime}$ & $56.53( \pm 9.93)$ & $56.12( \pm 1.47)$ & $60.03( \pm 13.40)$ & $57.95( \pm 4.16$ \\
$(\mathrm{GPa})$ & $C_{13}^{\prime \prime}$ & & $1.25( \pm 0.55)$ & $/$ & $1.55( \pm 0.50)$ \\
$C_{22}$ & $C_{22}^{\prime}$ & $109.31( \pm 3.04)$ & $108.13( \pm 1.45)$ & $110.88( \pm 21.18)$ & $108.35( \pm 2.56)$ \\
$(\mathrm{GPa})$ & $C_{22}^{\prime \prime}$ & $/$ & $1.72( \pm 0.28)$ & $/$ & $3.05( \pm 0.96)$ \\
$C_{23}$ & $C_{23}^{\prime}$ & $56.53( \pm 9.93)$ & $56.12( \pm 1.47)$ & $59.45( \pm 11.55)$ & $57.5( \pm 3.39)$ \\
$(\mathrm{GPa})$ & $C_{23}^{\prime \prime}$ & $/$ & $1.25( \pm 0.55)$ & $/$ & $1.45( \pm 0.46)$ \\
$C_{33}$ & $C_{33}^{\prime}$ & $109.31( \pm 3.04)$ & $108.13( \pm 1.45)$ & $119.74( \pm 7.59)$ & $111.91( \pm 1.83)$ \\
$(\mathrm{GPa})$ & $C_{33}^{\prime \prime}$ & $/$ & $1.72( \pm 0.28)$ & $/$ & $0.11( \pm 0.01)$ \\
$C_{44}$ & $C_{44}^{\prime}$ & $26.39( \pm 6.84)$ & $26.01( \pm 0.13)$ & $26.42( \pm 5.67)$ & $25.42( \pm 1.65)$ \\
$(\mathrm{GPa})$ & $C_{44}^{\prime \prime}$ & $/$ & $0.24( \pm 0.02)$ & $/$ & $0.63( \pm 0.23)$ \\
$C_{55}$ & $C_{55}^{\prime}$ & $26.39( \pm 6.84)$ & $26.01( \pm 0.13)$ & $25.63( \pm 5.95)$ & $25.29( \pm 1.98)$ \\
$(\mathrm{GPa})$ & $C_{55}^{\prime \prime}$ & $/$ & $0.24( \pm 0.02)$ & $/$ & $0.64( \pm 0.24)$ \\
$C_{66}$ & $C_{66}^{\prime}$ & $26.39( \pm 6.84)$ & $26.01( \pm 0.13)$ & $28.98( \pm 8.70)$ & $27.67( \pm 13.09)$ \\
$(\mathrm{GPa})$ & $C_{66}^{\prime \prime}$ & $/$ & $0.24( \pm 0.02)$ & $/$ & $0.86( \pm 0.49)$ \\
$\phi_{e}$ & $/$ & $/$ & $/$ & $-11.94^{\circ}\left( \pm 46.89^{\circ}\right)$ & $-22.00^{\circ}\left( \pm 46.90^{\circ}\right)$ \\
\hline & & & &
\end{tabular}

viscosity tensor. This is evidenced by the viscosity tensor which -by necessitybreaks the isotropic symmetry to allow a better fit, and is further illustrated in the cross-sectional plot of Figure 10 where a better match between the experiment and the forward simulation is noticed for the orthotropic assumption 


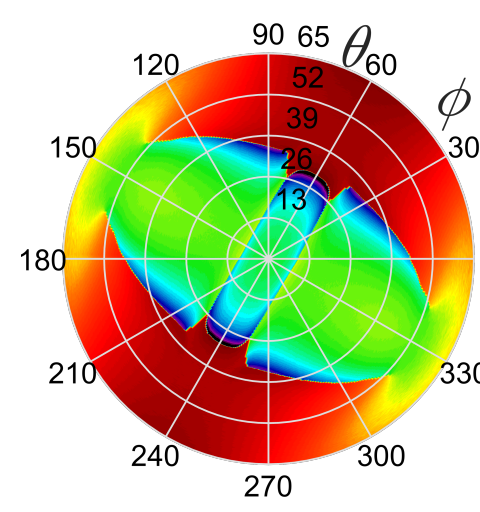

(a)

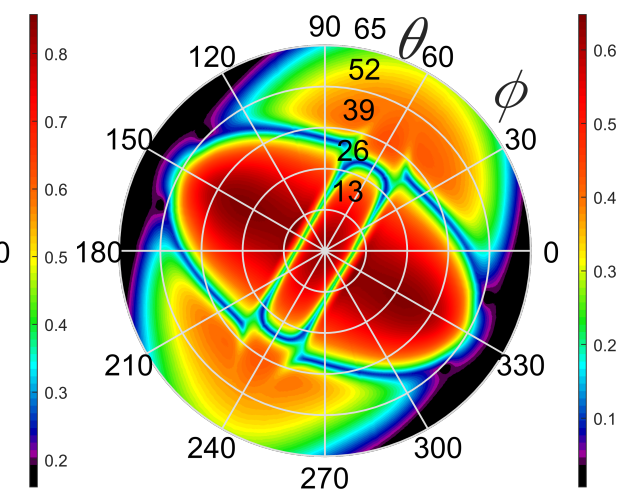

(b)

Figure 11: TOF P-UPS plot(a) and amplitude P-UPS plot(b) corresponding to a measurement on a unidirectional $[0]_{4} \mathrm{C} / \mathrm{E}$ plate $(d=1.200 \pm 0.006 \mathrm{~mm})$. The TOF colorbar is represented in $\mu$ s. The amplitude colorbar is normalized to the maximum amplitude of the input signal.

(no a-priori knowledge). The mismatch for the isotropic symmetry assumption at small incidence angles (prior to the first minimum) can be attributed to the viscosity of the $C_{33}$ parameter, which is a factor of 15 larger compared to the orthotropic assumption. However, a decrease in the viscosity of the $C_{33}$ parameter implicates a reduction in the material attenuation over the entire range of the incidence angles, subsequently increasing the maximum transmitted amplitude. This larger amplitude has to be compensated for by increasing the viscosity of the other parameters. The amplitude spike near the Rayleigh wave $\left(\theta=30^{\circ}\right)$, present in the isotropic assumption is attributed to low viscosity values for the $C_{44}, C_{55}$ and $C_{66}$ viscosity and has to be increased (factor of 3 ) to match the experimental P-UPS recording.

\subsection{Characterization for a carbon/epoxy plate based on real experimental P- UPS data}

The third and last case study of this paper deals with the inversion of the material properties for a unidirectional $[0]_{4}$ carbon/epoxy plate based on experimental P-UPS data. The plate has a thickness $d=1.200 \pm 0.006 \mathrm{~mm}$, density $\rho=1528 \mathrm{~kg} / \mathrm{m}^{3}$ and a fiber volume fraction Vf of $55 \%$. This sample has been 


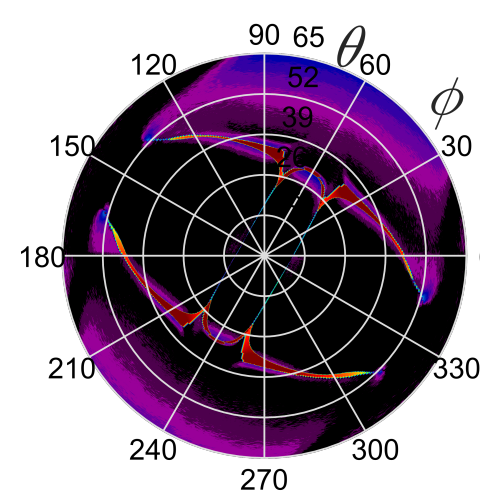

(a)

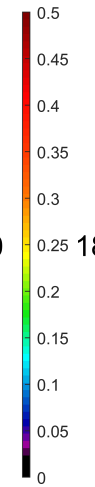

0

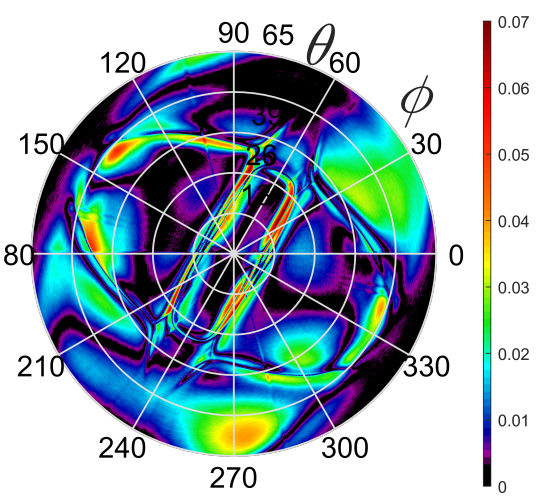

(b)

Figure 12: Difference TOF P-UPS (a) and Difference amplitude P-UPS (b) between the experimental polar plot for a unidirectional $[0]_{4}$ carbon/epoxy plate and the forward simulations based on the results of the inversion procedure. The TOF colorbar is represented in $\mu$ s. The amplitude colorbar is normalized to the maximum amplitude of the input signal.

autoclave manufactured according to the specification of the supplier. In the perfect case (perfectly aligned fibers and lay-up), one expects that the sample belongs to the transversal isotropic symmetry class with 5 independent tensor coefficients. In reality, the autoclave pressure cycle, the manual layer stacking and local distortions in the prepreg material may cause a deviation from the expected transverse isotropic symmetry. Therefore, no assumptions on the symmetry is taken a priori, and a general orthotropic symmetry class is considered for the inversion procedure. The experimentally obtained TOF and amplitude P-UPS plots are shown in Figures $11 \mathrm{a}$ and $11 \mathrm{~b}$ respectively. An initial visual inspection clearly reveals the anisotropic nature of the carbon/epoxy plate and the presence of a deviation angle $\phi_{e}$ in the neighborhood of $-30^{\circ}$.

\section{Stage I: inversion of the elastic tensor}

Identical bounds as in the first test-case are chosen for the elasticity search space. The results for the stage I inversion are given in the third column of Table 3 and yield values within expectation for a carbon/epoxy laminate. The in-plane deviation angle $\phi_{e}$ is found to equal $-29.81 \pm 0.34^{\circ}$ which is in good 
agreement with the visual estimate $\left(\approx 30^{\circ}\right)$. The difference TOF polar plot in Figure $12 \mathrm{a}$ clearly shows that although the overall deviation is low (TOF mismatch below $0.05 \mu \mathrm{s})$ there are still zones with large errors $(>0.1 \mu \mathrm{s})$. These errors are mainly situated in the vicinity of the characteristic contours and can again be attributed to the mismatch factors mentioned in the earlier cases.

\section{Stage II: viscoelastic characterization}

The results following the second stage of the inversion are summarized in the fourth column of Table 3 and the match between the experimental polar plot and prediction based on the inversion parameters is visualized by a difference amplitude polar plot in Figure 12b. A comparison of the associated elastic engineering constants (in the notation $E_{i i}, \nu_{i j}$ and $G_{i j}$ ) with values obtained and estimated from literature [24 26] (last three columns of Table 3) reveals that the inverted elasticity constants are within the expected range for a unidirectional carbon-epoxy material. In the study of Degrieck [24, these engineering constants are obtained through tensile tests of a UD C/E plate with a similar fiber volume fraction $V_{f}$ of approximately $55 \%$ as used here. Italic values represent estimations of parameters which cannot be determined by the experimental technique. The elasticity values taken from the studies of Sevenois [25] and Soden [26] are respectively determined through contact ultrasound and indirect measurements (e.g. biaxial tests) for a UD C/E plate with $V_{f}$ of $60 \%$. The $E_{i i}$ values in the latter studies are consistently higher, whereas the values for $G_{i j}$ tend to match well. The complementary viscosity constants that can be inferred with our two-stage inversion approach display values that are within the reasonable range of $2 \%$ to $8 \%$ of the elastic constants [1, 3, 27]. Additionally, the inverted in-plane deviation angle was found to be $-30.06 \pm 0.01^{\circ}$, which seems a highly accurate result given the small error bounds. Figure 12 confirms that the inverted values form a good representation of the investigated material as the deviations shown in the difference plot are well below $0.05 \mathrm{~V}$ over most of the incidence angles $\Psi$, aside from small zones with larger errors $(>0.05 \mathrm{~V})$ around the characteristic contours. These divergences have a similar nature 
Table 3: Viscoelastic C-tensor inversion results for stage I and II based on a P-UPS experiment performed on a unidirectional $[0]_{4}$ carbon/epoxy plate of thickness $d=1.200 \pm 0.006 \mathrm{~mm}$ and density $\rho=1528 \mathrm{~kg} / \mathrm{m}^{3}$. The transformation to engineering constants is obtained with the output of stage II. The last three columns represent literature C-tensor measurement values on UD $\mathrm{C} / \mathrm{E}$ materials. Italic values are estimated parameters.

\begin{tabular}{llll|llllll}
\hline & & Output stage I & Output stage II & Engineering & P-UPS values & Degrieck $[24]$ & Sevenois $[25]$ & Soden $[26]$ \\
\hline$C_{11}$ & $C_{11}^{\prime}$ & $118.76( \pm 20.3)$ & $113.24( \pm 3.49)$ & $E_{1}$ & $E_{1}^{\prime}$ & $108.87( \pm 2.56)$ & 119.13 & $135.37( \pm 1.08)$ & 138.00 \\
$(\mathrm{GPa})$ & $C_{11}^{\prime \prime}$ & $/$ & $7.89( \pm 1.54)$ & $(\mathrm{GPa})$ & $E_{1}^{\prime \prime}$ & $6.51( \pm 1.36)$ & $/$ & $/$ & $/$ \\
$C_{12}$ & $C_{12}^{\prime}$ & $7.29( \pm 1.32)$ & $6.86( \pm 0.51)$ & $E_{2}$ & $E_{2}^{\prime}$ & $9.61( \pm 0.09)$ & 8.85 & $11.36( \pm 0.24)$ & 11.00 \\
$(\mathrm{GPa})$ & $C_{12}^{\prime \prime}$ & $/$ & $0.64( \pm 0.08)$ & $(\mathrm{GPa})$ & $E_{2}^{\prime \prime}$ & $0.42( \pm 0.03)$ & $/$ & $/$ & $/$ \\
$C_{13}$ & $C_{13}^{\prime}$ & $6.73( \pm 2.31)$ & $6.46( \pm 1.14)$ & $E_{3}$ & $E_{3}^{\prime}$ & $10.04( \pm 0.06)$ & 8.85 & $11.53( \pm 0.29)$ & 11.00 \\
$(\mathrm{GPa})$ & $C_{13}^{\prime \prime}$ & $/$ & $0.58( \pm 0.06)$ & $(\mathrm{GPa})$ & $E_{3}^{\prime \prime}$ & $0.31( \pm 0.01)$ & $/$ & $/$ & $/$ \\
$C_{22}$ & $C_{22}^{\prime}$ & $12.13( \pm 2.28)$ & $13.21( \pm 0.10)$ & $G_{23}$ & $G_{23}^{\prime}$ & $3.29( \pm 0.02)$ & 3.00 & $3.23( \pm 0.05)$ & 3.93 \\
$(\mathrm{GPa})$ & $C_{22}^{\prime \prime}$ & $/$ & $0.49( \pm 0.04)$ & $(\mathrm{GPa})$ & $G_{23}^{\prime \prime}$ & $0.15( \pm 0.02)$ & $/$ & $/$ & $/$ \\
$C_{23}$ & $C_{23}^{\prime}$ & $6.48( \pm 1.4)$ & $6.92( \pm 0.29)$ & $G_{13}$ & $G_{13}^{\prime}$ & $5.15( \pm 0.02)$ & 5.50 & $5.40( \pm 0.28)$ & 5.50 \\
$(\mathrm{GPa})$ & $C_{23}^{\prime \prime}$ & $/$ & $0.11( \pm 0.03)$ & $(\mathrm{GPa})$ & $G_{13}^{\prime \prime}$ & $0.30( \pm 0.01)$ & $/$ & $/$ & $/$ \\
$C_{33}$ & $C_{33}^{\prime}$ & $14.84( \pm 0.76)$ & $13.73( \pm 0.38)$ & $G_{12}$ & $G_{12}^{\prime}$ & $5.11( \pm 0.01)$ & 5.50 & $5.40( \pm 0.35)$ & 5.50 \\
$(\mathrm{GPa})$ & $C_{33}^{\prime \prime}$ & $/$ & $0.36( \pm 0.01)$ & $(\mathrm{GPa})$ & $G_{12}^{\prime \prime}$ & $0.30( \pm 0.01)$ & $/$ & $/$ & $/$ \\
$C_{44}$ & $C_{44}^{\prime}$ & $3.46( \pm 0.91)$ & $3.29( \pm 0.02)$ & $\nu_{12}$ & $\nu_{12}^{\prime}$ & $0.37( \pm 0.02)$ & 0.31 & $0.20( \pm 0.06)$ & 0.28 \\
$(\mathrm{GPa})$ & $C_{44}^{\prime \prime}$ & $/$ & $0.15( \pm 0.02)$ & () & $\nu_{12}^{\prime \prime}$ & $0.99( \pm 0.31)$ & $/$ & $/$ & $/$ \\
$C_{55}$ & $C_{55}^{\prime}$ & $5.20( \pm 0.73)$ & $5.15( \pm 0.02)$ & $\nu_{13}$ & $\nu_{13}^{\prime}$ & $0.28( \pm 0.07)$ & 0.31 & $0.17( \pm 0.06)$ & 0.28 \\
$(\mathrm{GPa})$ & $C_{55}^{\prime \prime}$ & $/$ & $0.30( \pm 0.01)$ & () & $\nu_{13}^{\prime \prime}$ & $1.30( \pm 0.34)$ & $/$ & $/$ & $/$ \\
$C_{66}$ & $C_{66}^{\prime}$ & $5.21( \pm 0.87)$ & $5.11( \pm 0.01)$ & $\nu_{23}$ & $\nu_{23}^{\prime}$ & $0.49( \pm 0.01)$ & 0.48 & $0.37( \pm 0.02)$ & 0.40 \\
$(\mathrm{GPa})$ & $C_{66}^{\prime \prime}$ & $/$ & $0.30( \pm 0.01)$ & () & $\nu_{23}^{\prime \prime}$ & $0.21( \pm 0.08)$ & $/$ & $/$ & $/$ \\
$\phi_{e}$ & $/$ & $-29.81^{\circ}\left( \pm 0.34^{\circ}\right)$ & $-30.06^{\circ}\left( \pm 0.03^{\circ}\right)$ & $\phi_{e}$ & $/$ & $-30.06^{\circ}\left( \pm 0.03^{\circ}\right)$ & $/$ & $/$ & $/$ \\
\hline
\end{tabular}

as explained in the previous section. Regardless of these small deviations, it can be concluded that the results of this study demonstrate that the two-stage approach is effectively able to determine the full viscoelastic properties of any given material having at least orthotropic symmetry. 


\section{Conclusion}

An enhanced two-stage optimization procedure on the basis of the Pulsed Ultrasonic Polar Scan (P-UPS) is introduced for the determination of the viscoelastic properties of orthotropic plates. The optimization procedure uses a particle swarm optimization approach to search for the optimal parameter set in a 19-dimensional parameter search space: i.e. 9 parameters for the elastic tensor, 9 parameters for the viscous tensor and 1 parameter for the main axis of orthotropy. The first stage of the optimization procedure exploits the TOF P-UPS landscape to obtain good estimates for the orthotropic elastic tensor and the main axes of orthotropy. The second stage of the optimization procedure uses the amplitude P-UPS data, combined with the output from stage I, in order to determine the full orthotropic viscoelastic tensor. The procedure has been first validated on synthetic data yielding an excellent agreement between targeted and the inverted complex stiffness tensors, with relative errors on the elastic tensor below $0.5 \%$ and on the viscosity tensor below $2 \%$. The optimization procedure has further been applied on experimental P-UPS for an aluminum plate as well as for a $[0]_{8}$ carbon/epoxy laminate. The inversion results for the aluminum are in close agreement with values reported in literature, and show the robustness to postulations about symmetry classes. The optimized stiffness tensor for the unidirectional carbon/epoxy laminate is within the expectation values, and agrees well with partial datasets available from tensile tests.

\section{Acknowledgments}

The research leading to these results has gratefully received funding from the fund for scientific research-Flanders (FWO Vlaanderen, grant 1S45216N, G0B9515N and $12 \mathrm{~T} 5418 \mathrm{~N}$ ) and the NVIDIA corporation. 


\section{References}

[1] Bernard Hosten. Reflection and transmission of acoustic plane waves on an immersed orthotropic and viscoelastic solid layer. The Journal of the Acoustical Society of America, 89(6):2745-2752, 1991.

[2] P.W.A. Stijnman. Determination of the elastic constants of some composites by using ultrasonic velocity measurements. Composites, 26(8):597-604, 1995.

[3] B Hosten, M Deschamps, and B R Tittmann. Inhomogeneous wave generation and propagation in lossy anisotropic solids. Application to the characterization of viscoelastic composite materials. The Journal of the Acoustical Society of America, 82(5):1763-1770, 1987.

[4] S I Rokhlin and W Wang. Double through transmission bulk wave method for ultrasonic phase velocity measurement and determination of elastic constants of composite materials. Acoustical Society of America, 91(6):3303$3312,1992$.

[5] S. I Rokhlin and W Wang. Critical angle measurement of elastic constants in composite material. The Journal of the Acoustical Society of America, 86(5):1876, 1989.

[6] Victor Munoz, Marianne Perrin, Marie-laetitia Pastor, Hélène Welemane, Arthur Cantarel, and Moussa Karama. Determination of the elastic properties in CFRP composites : comparison of different approaches based on tensile tests and ultrasonic characterization. Advances in Aircraft and Spacecraft Science, 2(3):249-261, 2015.

[7] Y. C. Chu, A.D. Degtyar, and S. I Rokhlin. On determination of orthotropic material moduli from ultrasonic velocity data in nonsymmetry planes. The Journal of the Acoustical Society of America, 95(6):3191, 1994. 
[8] Anna Castellano, Pilade Foti, Aguinaldo Fraddosio, Salvatore Marzano, and Mario Daniele Piccioni. Mechanical characterization of CFRP composites by ultrasonic immersion tests: Experimental and numerical approaches. Composites Part B: Engineering, 66:299-310, 2014.

[9] L Wang and S I Rokhlin. Ultrasonic wave interaction with multidirectional composites: modeling and experiment. The Journal of the Acoustical Society of America, 114(5):2582-2595, 2003.

[10] Arvid Martens, Mathias Kersemans, Jannes Daemen, Erik Verboven, Wim Van Paepegem, Joris Degrieck, S Delrue, and K Van Den Abeele. Numerical study of the time-of-flight pulsed ultrasonic polar scan for the determination of the full elasticity tensor of orthotropic plates. Composite Structures, 180:29-40, 2017.

[11] L Wang, A I Lavrentyev, and S I Rokhlin. Beam and phase effects in angle-beam-through-transmission method of ultrasonic velocity measurement. The Journal of the Acoustical Society of America, 113(3):1551-1559, 2003.

[12] CV White and Gray Iron. Metals handbook: Properties and selection: Irons, steels and high performance materials, vol. 1, 1990.

[13] M. Kersemans, A. Martens, N. Lammens, K. Van Den Abeele, J. Degrieck, F. Zastavnik, L. Pyl, H. Sol, and W. Van Paepegem. Identification of the Elastic Properties of Isotropic and Orthotropic Thin-Plate Materials with the Pulsed Ultrasonic Polar Scan. Experimental Mechanics, 54(6):1121$1132,2014$.

[14] Mathias Kersemans, Arvid Martens, Joris Degrieck, Koen Van Den Abeele, Steven Delrue, Lincy Pyl, Filip Zastavnik, Hugo Sol, and Wim Van Paepegem. The Ultrasonic Polar Scan for Composite Characterization and Damage Assessment: Past, Present and Future. Applied Sciences, 6(2):58, 2016. 
[15] M. Kersemans, A. Martens, K. Van Den Abeele, J. Degrieck, F. Zastavnik, L. Pyl, H. Sol, and W. Van Paepegem. Detection and Localization of Delaminations in Thin Carbon Fiber Reinforced Composites with the Ultrasonic Polar Scan. Journal of Nondestructive Evaluation, 33(4):522-534, 2014.

[16] Mathias Kersemans, Ives De Baere, Joris Degrieck, Koen Van Den Abeele, Lincy Pyl, Filip Zastavnik, Hugo Sol, and Wim Van Paepegem. Nondestructive damage assessment in fiber reinforced composites with the pulsed ultrasonic polar scan. Polymer Testing, 34:85-96, apr 2014.

[17] A. Marzani and L. De Marchi. Characterization of the elastic moduli in composite plates via dispersive guided waves data and genetic algorithms. Journal of Intelligent Material Systems and Structures, 24(17):2135-2147, 2012.

[18] Adnan H Nayfeh. Wave Propagation in Layered Anisotropic Media - with Applications to Composites, volume 39 of North-Holland Series in Applied Mathematics and Mechanics. Elsevier, 1995.

[19] Riccardo Poli, James Kennedy, and Tim Blackwell. Particle swarm optimization. Swarm intelligence, 1(1):33-57, 2007.

[20] Armand Wirgin. The inverse crime. arXiv.org, pages 1-10, 2004.

[21] Shivakumar I Ranganathan and Martin Ostoja-Starzewski. Universal elastic anisotropy index. Physical Review Letters, 101(5):055504, 2008.

[22] Roderic S Lakes. Viscoelastic Solids (1998). CRC press, 2017.

[23] Joris Degrieck. Analyse van impact op een vezelversterkte kunststof., 1990.

[24] R.D.B. Sevenois, D. Garoz, E. Verboven, S.W.F. Spronk, F.A. Gilabert, M. Kersemans, L. Pyl, and W. Van Paepegem. Multiscale approach for identification of transverse isotropic carbon fibre properties and prediction 
of woven elastic properties using ultrasonic identification. Composites Science and Technology, 168:160 - 169, 2018.

[25] P.D. Soden, M.J. Hinton, and A.S. Kaddour. Lamina properties, lay-up configurations and loading conditions for a range of fibre-reinforced composite laminates. Composites Science and Technology, 58(7):1011 - 1022, 1998.

[26] Marc Deschamps and Bernard Hosten. The effects of viscoelasticity on the reflection and transmission of ultrasonic waves by an orthotropic plate. The Journal of the Acoustical Society of America, 91(4):2007-2015, 1992. 